\title{
Parabolic flight: experiencing zero gravity to envisage the future of human evolution
}

\author{
Tetsuro Matsuzawa $a^{1,2,3}$
}

Published online: 6 December 2017

(C) Japan Monkey Centre and Springer Japan KK, part of Springer Nature 2017

I experienced parabolic flight for the first time recently. In space, people and objects appear weightless. The state of weightlessness can be simulated for a very short time within the Earth's atmosphere inside an aircraft. This is achieved by carrying out a parabolic maneuver: the plane follows a large inverted parabola, steeply up then down, creating free fall inside around the apex of the parabola, simulating zero gravity (microgravity) for about $20 \mathrm{~s}$ continuously. I wanted to understand the psychological effects of zero gravity.

The Primate Research Institute of Kyoto University is located close to Nagoya Airport, where the opportunity for parabolic flight is available. It was on October 28, 2017 that a team of seven, two professors and five students of Kyoto University, flew over the Pacific Ocean. The flight consisted of successive repetitions of rapid upward flight followed by rapid downward flight. During this process, passengers can experience zero gravity.

I found weightlessness to induce a unique and interesting sensation. At first, your body floats upwards from the floor. You can move your hands and feet freely, but your body floats in midair. If you release a pen from your grip, it will remain floating, right in front of your eyes. If you close your eyes, you can no longer tell which direction is up or down, only relative to the orientation of your own body.

This research flight was a joint endeavor conducted with Dr. Takao Doi of Kyoto University. Dr. Doi is an astronaut who has taken part in two space shuttle missions. The first time he experienced space flight was in 1997 (STS87) as Mission Specialist; the second was in 2008 (STS123) as Chief Commander of the Japanese Experiment Module (JEM), named Kibo ("hope" in Japanese), of the

Tetsuro Matsuzawa

matsuzawa.tetsuro.8w@kyoto-u.ac.jp

1 Institute for Advanced Study, Kyoto University, Kyoto, Japan

2 Primate Research Institute, Kyoto University, Inuyama, Japan

3 Japan Monkey Centre, Inuyama, Japan
International Space Station (ISS) set up by the Japan Aerospace Exploration Agency (JAXA). Dr. Doi was the first Japanese person to "spacewalk", during STS-87. Wearing a space suit, he opened the airlock hatch of Space Shuttle Columbia and exited; he carried out extravehicular activity (EVA). Dr. Doi became a professor of Kyoto University in 2016 with the aim of establishing a new scientific discipline, Human Space Activities, at the Unit of Synergetic Studies for Space. According to Dr. Doi, the weightlessness that we experienced in the parabolic flight felt exactly the same as the zero gravity he experienced in outer space.

There is a key difference between achieving simulated zero gravity through parabolic flight and the zero gravity experienced on a space station in stable orbit. During a parabolic flight, you must undergo $2 \mathrm{~g}$ gravity during the rapid upward flight; an acceleration equivalent to twice the gravity experienced on the Earth's surface. Under the $2 g$ condition, it felt like I was being compressed down towards the floor. It was very hard to move my arms and hands. Then, after the $2 g$ condition ceased, I suddenly experienced zero gravity.

During the parabolic flight we carried out novel cognitive research. To assess cognitive performance under zero gravity conditions, compared with other levels of gravity, we invented a test battery of aero-cognitive tasks. An example temporal task was to measure the subjective experience of time while in space. We measured $10 \mathrm{~s}$ using a stopwatch. Each subject had to estimate $10 \mathrm{~s}$ using their internal body clock. The measurement was carried out three times at each of the following four conditions: before the $1 \mathrm{~g}$ flight; during zero gravity; at $2 g$; and after the flight at $1 \mathrm{~g}$. The accuracy of each estimation and overall variance were calculated.

An example spatial task was to ask which direction is "up", "ahead", and "down" when you have your eyes closed during zero gravity. Working memory ability was tested both before and after the flight. Hormonal changes caused by stress were measured using a kit to collect subjects' saliva. Hormonal changes were sampled at each phase of the flight. 
One team member wore a Tobii eye-tacker to document visual fixation points, reference points, during the flight. The entire process of the flight was recorded by a head-mounted video camera worn by each of the seven participants, providing a visual record of what they could see during the flight (Figs. 1, 2). In addition, two fixed-position video cameras recorded a wide-angle view showing the movement of the whole team in the aircraft.
After experiencing zero gravity a total of nine times, we then experienced gravity of $1 / 6 \mathrm{~g}$. This can be produced by changing the angle, speed, and other factors of the downward flight. Gravity of $1 / 6$ (or 0.165 ) $g$ is equivalent to being on the Moon. You can stand on the floor, but your body floats upwards if you push off lightly. It is something like a moon walk, taking steps very softly and gently.
Fig. 1 We experienced weightlessness on a parabolic flight
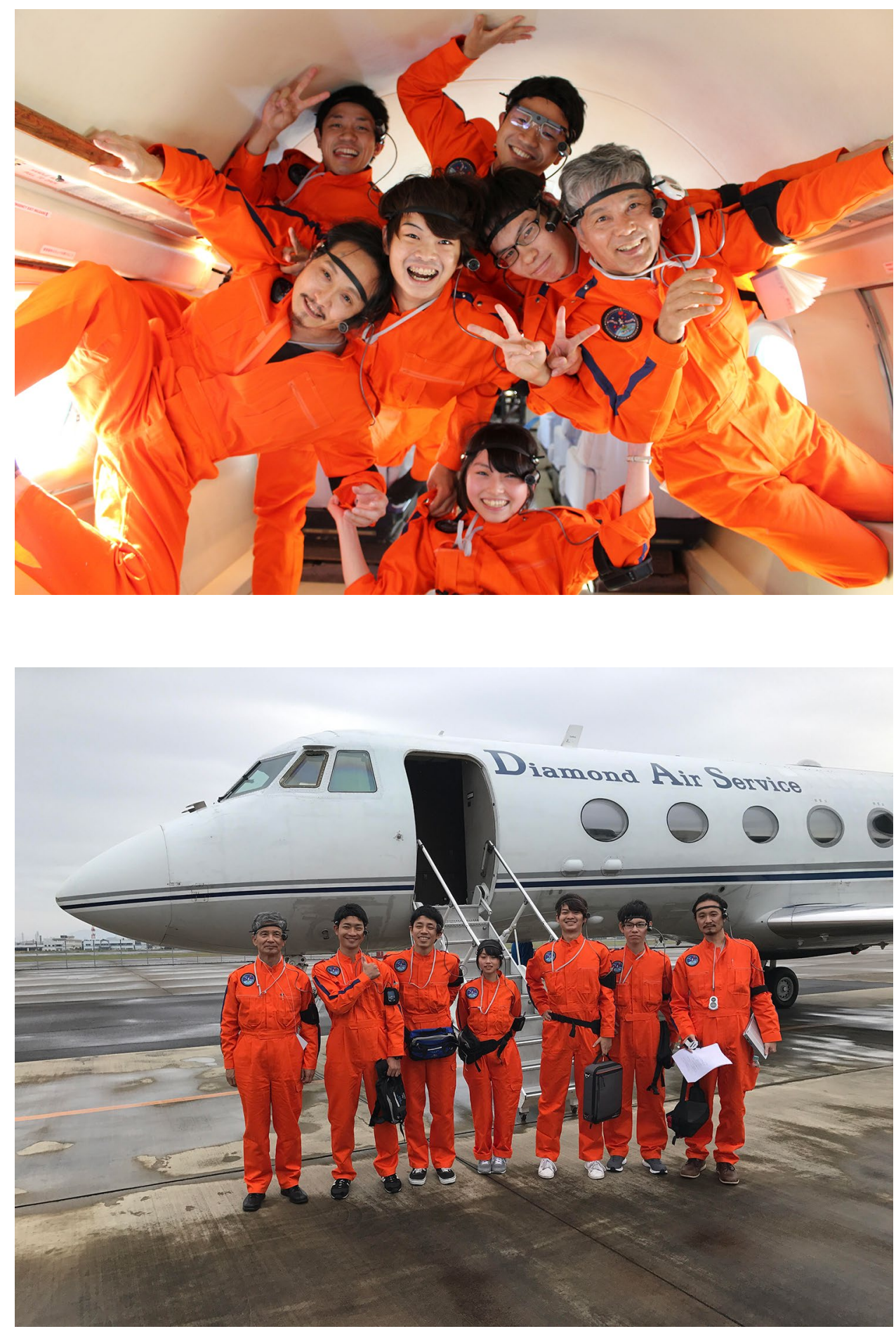

Fig. 2 The team members on the parabolic flight of October 28, 2017 
Thus, my team members and I experienced four different kinds of gravity in the course of a one parabolic flight: 0 , $1 / 6,1$, and $2 g$. I know, logically, that my body remains the same, of course, regardless of the change in gravity. However, the sensation in my body changed drastically along with the changing levels of gravity. In our daily life, we are not consciously aware of the action of gravity. The parabolic flight gave me the unique opportunity to feel gravity as a tangible, real force controlling my body.

In short, all of human history, events, and thoughts (excepting spaceflight) have so far happened in a world of more or less unchanging gravity at $1 \mathrm{~g}$. In the near future, humans will return to the Moon, go to Mars, and travel deeper into outer space. I recognize that in these new circumstances our sensations of our own bodies may change because of the changes in the environment in terms of gravity. This may result not only in changes to the sensations of the body but also to the mind in general.

I have researched Primatology for decades. Primatology is a discipline that encompasses the study of primates, including humans, through a comparative approach to understand human evolution. What is human nature? Where did we come from? How did we get here? In sum, these are all questions about our evolutionary history. In contrast to these questions about our past, we should now ask another question about our evolutionary future: where will we go? I want to reach into the future. I want to know how humans might evolve. That was the primary motivation for me to try out parabolic flight. It is actually the first step towards the Moon. When I gained a place at Kyoto University at 18 years of age, humans had just reached the Moon. The Apollo 11 mission sent astronauts to the Moon. Since then, no one has reached the moon. Our generation will reach the Moon and may even inhabit the moon, that is, a new environment with gravity of $1 / 6 \mathrm{~g}$.

Suppose that you lived on the Moon. From there, you would see Earth, just as we can see the Moon from the Earth. A naïve way of thinking may lead you to expect to see the
Earth rising then setting below the horizon; however, this is completely wrong. The Earth does not move at all when observed from your site on the Moon. The Earth is always located at one particular point in the night sky as seen from the Moon, determined by your location; the Earth appears stationary. The Earth does not move at all and changes in shape, to the observer, just like the Moon waxing and waning as viewed from the Earth. On the Moon, there is a long night, lasting 2 weeks, and a similarly long daytime of 2 weeks. The gravity is $1 / 6 \mathrm{~g}$, i.e., one-sixth that of the Earth. The environment on the Moon is quite different from what humans have experienced on planet Earth over the past millions of years.

Human ancestors dwelled in the forest and invaded a new environmental niche, the open savanna land. Adaptation to the new environment shaped human nature: the human body, mind, and way of life. If so, how will humans evolve in new future environments such as the Moon? To look for clues to our future, I believe that parabolic flight and the experience of weightlessness may provide us with new insight for understanding possible paths of future evolution. I also wish to see the Earth from the Moon. Seeing our planet from outer space will surely bring us a new perspective and understanding of ourselves.

This December, we will take a second parabolic flight with seven team members and two further flights in 2018. Parabolic flight is the first step for Primatology in the future. Taking an entirely novel and pioneering perspective, as no one has before me, I dream about the future of Primatology.

Acknowledgements Thanks are due to Prof. Juichi Yamagiwa, President of Kyoto University and former Editor-in-Chief of the journal Primates, for giving us the financial support for our parabolic flight. Another financial support is from MEXT specially-promoted research grant $16 \mathrm{H} 06283$ to the author. I appreciate the guidance provided by Prof. Takao Doi. 